PREMIÈRE PARTIE

\title{
Les contraintes
}


7 KIVSDJHIQQMQWRQDQIOIKEOQN 
CHAPITRE 1

\section{Les évolutions \\ démographiques}

HENRI LERIDON 
7 KIVSDJHIQQMQWRQDQIOIKEOQN 


\section{Les contraintes démographiques}

La plupart des études sur les conséquences des changements climatiques éventuels, des besoins en ressources alimentaires ou en énergie à l'horizon 2050 ou 2100, s'appuient sur les projections de population produites par les organismes internationaux, le plus souvent celles de la Division de la population aux Nations unies. On retient ainsi très habituellement le chiffre de " 9 milliards d'habitants en 2050 ». C'est en effet l'ordre de grandeur de la projection centrale (medium scenario) de cet organisme, qui prévoit 9,1 milliards dans ses toute dernières projections (base 2008). Les estimations proposées par d'autres organismes ne s'éloignent pas beaucoup de ce chiffre, et il existe aussi un consensus pour penser qu'un maximum pourrait être atteint autour de 9-10 milliards peu après 2050 .

Il est cependant légitime de ne pas prendre pour argent comptant ces estimations. Après tout, il est arrivé (aussi) aux démographes de se tromper dans le passé, et un écart de plus ou moins un milliard ne saurait être considéré comme négligeable. Nous allons donc brièvement rappeler la méthodologie des projections démographiques, les sources d'incertitude et le degré de précision des résultats proposés.

\subsection{Méthodes et valeur des projections démographiques}

Une projection de la population mondiale consiste à suivre, année après année, l'évolution de la population de chaque pays du monde, par sexe et âge, compte tenu des hypothèses faites pour ce pays en termes de fécondité, mortalité et migrations, puis à agréger au niveau régional et mondial les résultats obtenus. Les risques d'erreur sont de deux ordres : soit sur les effectifs ou les autres paramètres initiaux relatifs aux divers pays, soit sur la vraisemblance des hypothèses retenues.

Il existe en effet toujours une incertitude sur la population initiale. On l'estime à $2 \%$ pour la population mondiale, et la précision n'est pas meilleure à l'échelle de chaque pays : on donne un taux d'incertitude à peu près égal pour la population française, par exemple, l'estimation de l'erreur étant basée sur une opération de contrôle du recensement. Il arrive que l'on doive réviser fortement, dans un sens ou dans l'autre, la population d'un pays après un recensement: la population chinoise avait été révisée à la hausse de plus de 100 millions en 1953. D'autres "surprises », de moindre ampleur, ont été observées dans divers pays : ainsi, la population « officielle » du Nigeria est-elle passée de 122 à 
88 millions en 1991, soit une perte relative de $28 \%$... Dans l'ensemble, néanmoins, l'incertitude a diminué dans les décennies récentes grâce aux efforts déployés pour réaliser des recensements convenables un peu partout. Les niveaux de la fécondité sont assez bien connus, par les statistiques de l'état civil là où celui-ci existe, par des enquêtes démographiques ailleurs. Ceux de la mortalité sont moins bien connus, les enquêtes suppléant plus difficilement l'absence de données d'état civil. Il existe toutefois des méthodes de comptage des décès ou d'estimations indirectes qui ne reposent pas sur un enregistrement de type état civil.

Le second risque d'erreur réside dans le choix des hypothèses d'évolution pour la fécondité, la mortalité et les migrations. Pour la mortalité, on extrapole l'espérance de vie initiale en se guidant sur l'expérience des pays plus avancés dans leur évolution. l'hypothèse de " catastrophes 》, voire de simples reculs qui pourraient résulter d'une détérioration des systèmes de soins (comme on en a observé en Russie et dans d'autres pays de l'ex-Union soviétique) n'est pas envisagée; seul le sida fait l'objet d'un traitement spécifique dans les projections onusiennes. Pour la fécondité, on procède un peu de la même façon, avec une difficulté particulière quand la baisse n'est pas du tout commencée ( $c$ 'est le cas dans une douzaine de pays, presque tous en Afrique subsaharienne) : il faut alors décider d'une date de début de baisse, sans éléments déterminants pour guider ce choix. Mais ces situations ne concernent qu'environ $3 \%$ de la population mondiale. Pour les migrations, enfin, les Nations unies retiennent des hypothèses prudentes, avec des flux annuels nets plutôt inférieurs à ceux observés dans la période récente; nous y reviendrons mais, évidemment, ces hypothèses sont sans effet au niveau de la population mondiale.

Reste un point important : le choix de l'horizon de la projection. C'est habituellement 2050, en particulier dans les projections proposées par les Nations unies tous les deux ans. Mais l'exercice a été parfois poussé jusqu'en 2100, voire 2150 et même 2300 dans une étude récente dont l'utilité nous parait bien maigre. En effet, il faut comprendre que dans une projection :

- à l'horizon 25 ans : l'évolution de la mortalité peut être assez bien prévue, et la part de la population à naître après la date de départ to (part qui est plus difficile à estimer en raison des incertitudes sur la fécondité) est limitée ;

- à 50 ans : plus de la moitié des effectifs dépend des hypothèses de fécondité, qu'il faut déjà appliquer à deux générations successives (une femme née en $t_{0}+20$ peut avoir des enfants à partir de $\left.t_{0}+40\right)$;

- au-delà : on ne sait plus gérer convenablement les hypothèses. Un écart très limité sur la fécondité, par exemple, a des conséquences énormes au bout de 100 ans (au moins 3 générations de naissances successives 
sont concernées!). Du coup, on décide généralement de "geler » les hypothèses à des niveaux assurant une croissance proche de zéro, et la projection perd tout intérêt.

En conclusion, on dispose aujourd'hui de bons outils pour faire des projections raisonnables à 30-50 ans, si les données initiales sont de bonne qualité et si des hypothèses réalistes peuvent être posées pour l'évolution des variables comportementales pendant cette période. La projection peut éventuellement être prolongée sur quelques dizaines d'années, à paramètres constants, pour mesurer les effets de l'inertie démographique encore présente après 50 ans.

\subsection{Les projections actuelles et leurs variantes}

Après un mauvais départ (en 1950 et 1954), largement excusable par la quantité et la qualité limitées des données disponibles, les projections successives de l'ONU ont été rapidement capables d'encadrer l'évolution réelle entre les hypothèses " haute » et " basse ». Pour l'hypothèse centrale, l'écart (excédent) à la valeur observée a été inférieur à $4 \%$ depuis 1978, et la valeur observée se trouvait bien plus proche de la projection centrale que des autres.

Sur quoi reposent les variantes haute et basse proposées? Essentiellement sur des hypothèses alternatives pour la fécondité. En pratique, les données le plus largement diffusées combinent le scénario central de mortalité avec quatre scénarios de fécondité :

- une hypothèse centrale (medium), dans laquelle la fécondité de chaque pays est supposée tendre vers une valeur commune, valeur atteinte le plus souvent avant 2050 ;

- une hypothèse de fécondité plus forte (high);

- une hypothèse de fécondité moins élevée (low);

- I'hypothèse d'une fécondité restant, dans chaque pays, au niveau observé en début de projection (constant).

Dans les dernières projections, la fécondité limite de l'hypothèse centrale est 1,85 enfant; l'hypothèse " haute » consiste à augmenter cette valeur limite en fin de période de 0,5 enfant par femme, et l'hypothèse « basse » à la diminuer d'autant. Ces écarts peuvent sembler faibles, mais ils génèrent de grosses différences dans l'effectif de la population mondiale en 2050 : celle-ci se situerait entre 8,0 et 10,5 milliards selon ces deux hypothèses (figure 1.1). Avec une fécondité inchangée de 2,6 enfants par femme (valeur actuelle de la moyenne mondiale), 


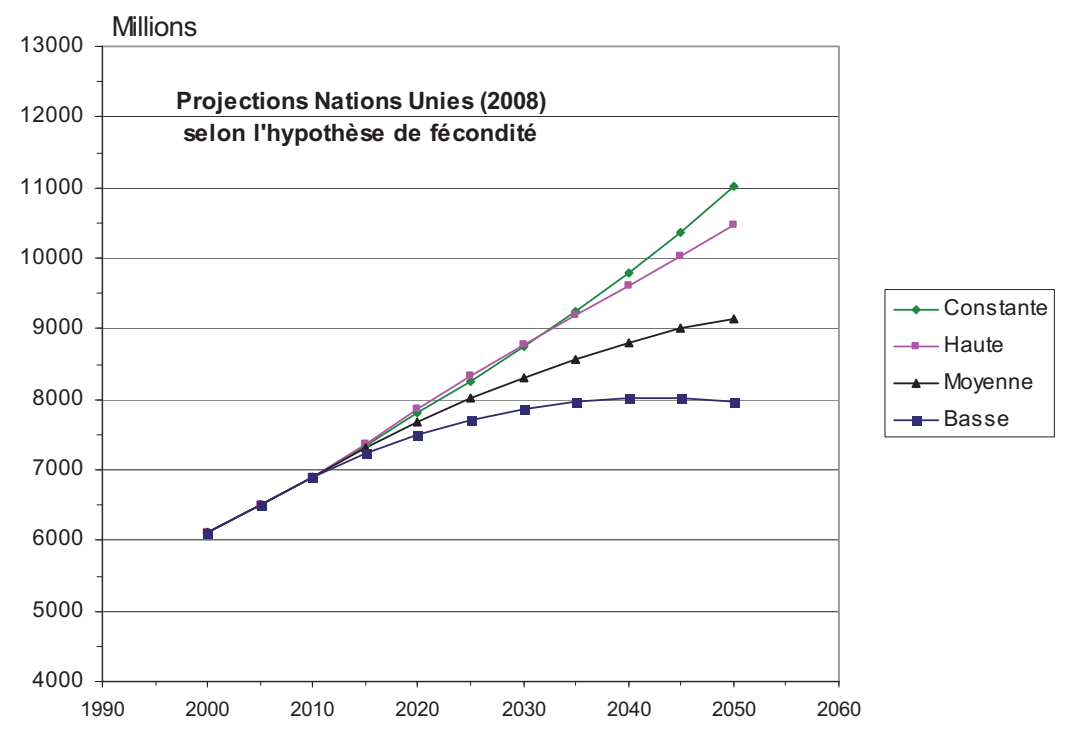

Figure 1.1

Projections démographiques 2000-2050 (Nations unies, 2008).

la population atteindrait 11,0 milliards en 2050. Mais en fait, comme nous l'avons dit, l'analyse des projections réalisées depuis les années 1960 montre que la valeur observée s'est toujours située assez près de l'hypothèse centrale.

On pourrait imaginer une réduction de la fécondité un peu moins rapide que prévu, mais la réalisation de l'hypothèse " haute » représenterait une erreur de prévision énorme sur la fécondité (2,5 enfants en 2050 au lieu de 2,0). De même, la réalisation intégrale de l'hypothèse basse supposerait que la fécondité s'établisse autour de 1,3 enfant par femme en Inde et en Chine comme en Europe ou dans les Amériques, et un peu en dessous de 2 en Afrique subsaharienne (5,4 actuellement). À quelles conditions peut-on envisager une baisse aussi rapide? L'exemple chinois a montré qu'une politique de quasi-coercition pouvait avoir des résultats. C'est en effet dans les années 1970-1978, celles de la mise en œuvre de la politique "wan, xi, shao" (naissances plus tardives, plus espacées, moins nombreuses), que la baisse a été la plus spectaculaire, la fécondité passant de 5,7 à 2,7 enfants par femme en 9 ans! La politique de "l'enfant unique », implémentée en 1979, n'a fait que poursuivre cette tendance à un rythme plus lent, le niveau actuel de la fécondité chinoise étant de l'ordre de 1,7 à 1,8 enfant. Ces résultats ont été obtenus au prix d'une mise sous surveillance étroite de la population (fixation de quotas de naissances à respecter au plan local, par exemple), et particulièrement des femmes (dont les grossesses, et même les menstruations, sont contrôlées dans les usines). En Chine, la pratique 
contraceptive n'est pas un droit, mais un devoir, et une grossesse « illégale » doit être interrompue.

Des résultats significatifs peuvent cependant être obtenus sans actions aussi contraignantes de la part de l'État. En Iran, par exemple, la fécondité a aussi chuté de moitié en 9 ans, passant de 5,3 en 1989 à 2,6 en 1998 (2,0 aujour$\mathrm{d}^{\prime}$ hui). Or un programme de diffusion de la contraception, non contraignant, avait été lancé fin 1989 par les autorités politiques, et les enquêtes ont confirmé la forte augmentation des pratiques contraceptives au cours des années 1990. II faut cependant noter que la fécondité avait déjà sensiblement baissé antérieurement, puisqu'elle était voisine de 7 enfants au début des années 1980 : d'autres facteurs étaient donc en jeu, parmi lesquels le décollage économique du pays et les progrès de la scolarisation. Leur effet s'est certainement poursuivi dans les années 1990.

Une réduction rapide de la fécondité là où elle est encore forte n'est donc pas impossible. Si l'on exclut les politiques coercitives, elle suppose surtout des évolutions culturelles majeures : plus la fécondité approche le seuil de 2 enfants, et a fortiori pour passer en-dessous, plus la détermination des femmes doit être forte pour surmonter les derniers obstacles culturels (résistance des maris ou de la parenté, par exemple). Leur faciliter l'accès à des méthodes contraceptives efficaces est alors une condition nécessaire, mais non suffisante.

Notons, finalement, que, si l'hypothèse basse de fécondité n'était réalisée que dans les pays actuellement les plus avancés économiquement (Europe, Amérique du Nord), avec 1,3 enfant à I'horizon 2050, la population mondiale ne serait diminuée que de 133 millions en 2050, soit 1,5\%, par rapport à l'hypothèse centrale. Par comparaison, l'Afrique contribuerait à cette diminution pour 250 millions, et l'Asie pour près de 700 millions.

\subsection{Où les 3 milliards d'habitants supplémentaires vivront-ils?}

La croissance sera concentrée sur l'Asie, où la fécondité a déjà beaucoup baissé mais où l'inertie accumulée dans les décennies récentes reste élevée (sa population augmentera en 50 ans de 1,53 milliard, soit $+41 \%$, représentant $50 \%$ du total de la croissance mondiale), et en Afrique où la fécondité est encore forte $(+1,18$ milliard, soit $+144 \%$, représentant $39 \%$ de la croissance mondiale) (figure 1.2). L'Amérique latine augmentera de $40 \%$ (+ 208 millions) tout comme l'Amérique du Nord (+ $40 \%$ soit + 129 millions). La population européenne, quant à elle, est supposée diminuer légèrement (- 36 millions). 


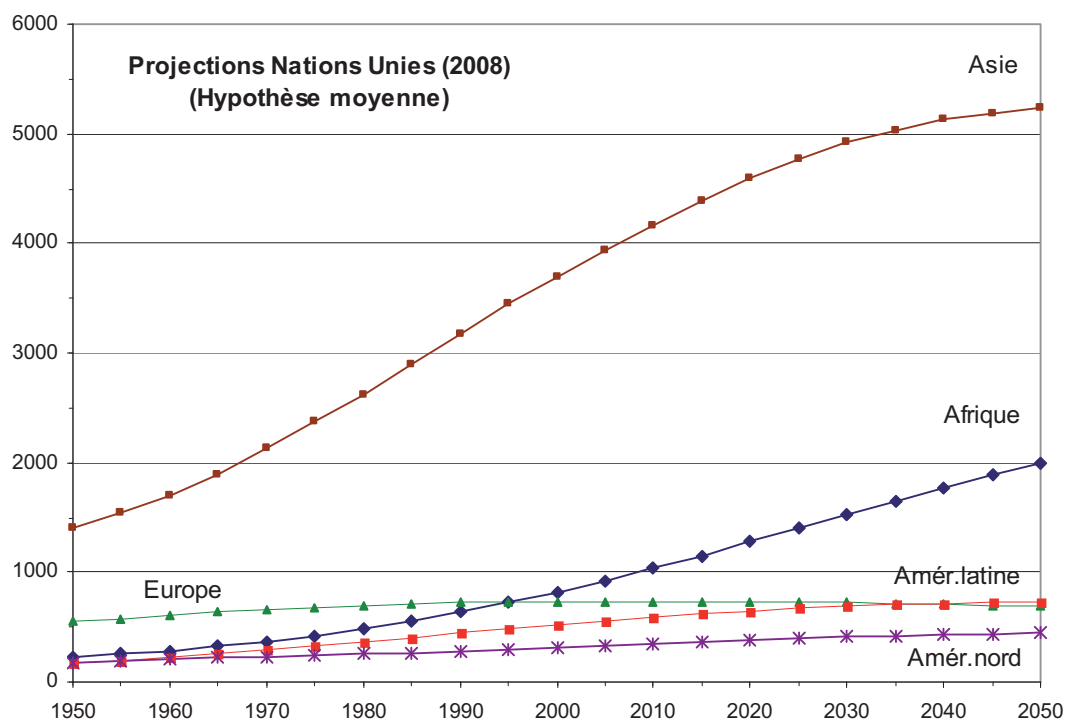

Figure 1.2

Populations des grandes régions du monde en 2000-2050 (Nations unies 2008, Hypothèse moyenne).

En Asie, la croissance concernera principalement le sous-continent indien (+ 890 millions, dont 600 en Inde). La fécondité a déjà beaucoup baissé en Inde : 2,7 enfants par femme aujourd'hui. Dans sept États (sur les 15 les plus peuplés), la fécondité est inférieure à 2,5 enfants par femme, avec un minimum au Kérala $(1,8)(V e ́ r o n, 2006)$. Dans cinq autres États, en revanche, elle dépasse encore 3 enfants (avec un maximum au Bihar : 4,3) : à l'évidence, les évolutions qui expliquent la basse fécondité des États du sud, comme le Kérala (alphabétisation, développement économique, bonne information sur les méthodes de régulation des naissances, baisse de la mortalité infantile...) tardent à produire tous leurs effets dans les États du nord. Mais, globalement, la forte croissance à venir de la population indienne doit beaucoup à un mécanisme $d^{\prime}$ inertie : même le Kérala, par exemple, affiche encore une croissance annuelle de $1 \%$. Les populations du Bangladesh (141 millions en 2000) et de l'Indonésie (205 millions) augmenteront chacune de 82 millions d'ici à 2050.

La Chine ajoutera 150 millions d'habitants au total mondial. La fécondité chinoise est déjà nettement inférieure à celle de l'Inde (environ 1,8 enfant actuellement), et comme la baisse de la fécondité a été plus rapide en Chine l'inertie démographique y est moindre. On a aussi assisté à une assez forte homogénéisation géographique des comportements, en dehors des grandes "municipalités » de la côte est (Shanghai, Beijing et Tianjin) où la fécondité ne dépasse pas 1,2 enfant par femme (Li Jianmin, 2002). C'est là, en effet, que la politique 
de l'enfant unique a été réellement appliquée à grande échelle. Ailleurs, des exceptions sont tolérées (notamment pour les ethnies minoritaires dans les provinces de l'ouest et du nord) ; l'objectif des autorités chinoises n'est d'ailleurs pas d'aboutir à un taux de fécondité proche de 1 enfant au plan national, notamment en raison de l'aggravation du processus de vieillissement qui en résulterait.

En Afrique, la croissance concernera surtout l'Afrique subsaharienne, à l'ouest comme à l'est (+ 388 et +458 millions respectivement), mais la population d'Afrique du Nord et celle d'Afrique centrale augmenteront aussi sensiblement. C'est en Afrique que l'on trouve une douzaine de pays n'ayant pas encore connu de baisse de leur fécondité.

L'Europe perdra 36 millions d'habitants (sur 726), essentiellement dans la Fédération de Russie qui devrait voir sa population diminuer de $21 \%$.

On assistera donc à une "redistribution » internationale des populations, mais une autre évolution majeure sera la concentration urbaine. En effet, sur la période 2007-2050, la population des villes augmentera davantage que la population mondiale $(+3,1$ milliards contre $+2,5)$, et sa part dans la population totale passera de 49 à $70 \%^{1}$ (tableau 1.1). Comme pour la population totale, la croissance de la population urbaine se situera essentiellement dans les pays en développement (+ 3,0 milliards). À l'horizon 2015, si Tokyo restera de loin la mégalopole la plus peuplée (36 millions), trois autres métropoles dépasseront légèrement 20 millions (Bombay, Mexico et San Paolo) et deux autres en seront proches (New York et Dehli). Toutefois, la croissance des agglomérations de plus de 10 millions d'habitants ne représentera qu'une faible part de la croissance urbaine : le plus gros (près de la moitié) se fera dans des villes de moins de 500000 habitants, et une bonne partie (30\%) dans les villes de 1 à 5 millions d'habitants.

Cette forte croissance urbaine posera d'importants défis en termes de gouvernance : problèmes de logements, de sécurité alimentaire, d'accès à l'eau potable... La situation sera particulièrement critique dans les métropoles d'Afrique subsaharienne, dont beaucoup sont des villes nouvelles, sans activité économique particulière, et incapables dès lors de fournir à leurs population un accès aux services de base et des ressources en quantités suffisantes. Il faut aussi être conscient du fait que la population des grandes métropoles est supérieure à celle de nombreux États.

\footnotetext{
'Bien que l'estimation de la population urbaine soit difficile à vérifier, la notion de « ville » n'étant pas un concept statistique ayant une définition universelle.
} 


\begin{tabular}{|c|c|c|c|c|c|c|c|c|c|}
\hline \multirow[t]{2}{*}{ Région } & \multicolumn{5}{|c|}{ Population (milliards) } & \multicolumn{4}{|c|}{ Taux d'accroissement annuel (\%) } \\
\hline & 1950 & 1957 & 2007 & 2025 & 2050 & 1950-1975 & $1975-2007$ & $2007-2025$ & $2025-2050$ \\
\hline Monde & 2,54 & 4,08 & 6,67 & 8,01 & 9,19 & 1,90 & 1,54 & 1,02 & 0,55 \\
\hline Régions plus développées & 0,81 & 1,05 & 1,22 & 1,26 & 1,25 & 1,01 & 0,48 & 0,16 & $-0,04$ \\
\hline Régions moins développées & 1,72 & 3,03 & 5,45 & 6,75 & 7,95 & 2,26 & 1,84 & 1,19 & 0,65 \\
\hline \multicolumn{10}{|c|}{ Population urbaine } \\
\hline Monde & 0,74 & 1,52 & 3,29 & 4,58 & 6,40 & 2,89 & 2,42 & 1,84 & 1,33 \\
\hline Régions plus développées & 0,43 & 0,70 & 0,91 & 0,99 & 1,07 & 1,98 & 0,81 & 0,49 & 0,30 \\
\hline Régions moins développées & 0,31 & 0,82 & 2,38 & 3,59 & 5,33 & 3,88 & 3,35 & 2,27 & 1,58 \\
\hline \multicolumn{10}{|l|}{ Population rurale } \\
\hline Monde & 1,80 & 2,56 & 3,38 & 3,43 & 2,79 & 1,41 & 0,87 & 0,08 & $-0,82$ \\
\hline Régions plus développées & 0,39 & 0,35 & 0,31 & 0,26 & 0,17 & $-0,44$ & $-0,32$ & $-0,94$ & $-1,67$ \\
\hline Régions moins développées & 1,41 & 2,21 & 3,06 & 3,16 & 2,62 & 1,80 & 1,02 & 0,17 & $-0,70$ \\
\hline \multicolumn{10}{|l|}{$\begin{array}{l}\text { Population urbaine } \\
\text { (\% du total) }\end{array}$} \\
\hline Monde & 29,1 & 37,3 & 49,4 & 57,2 & 69,6 & & & & \\
\hline Régions plus développées & 52,5 & 67,0 & 74,4 & 79,0 & 86,0 & & & & \\
\hline Régions moins développées & 18,0 & 27,0 & 43,8 & 53,2 & 67,0 & & & & \\
\hline
\end{tabular}

Tableau 1.1

Urbanisation : projections 2007 des Nations unies. 


\subsection{Le problème des migrations}

Selon les chiffres de l'Organisation internationale pour les migrations (OIM), on dénombre aujourd'hui plus de 200 millions de migrants internationaux dans le monde, définis comme des personnes résidant dans un pays différent de celui où elles sont nées. Ces 200 millions de migrants représentent un peu moins de $3 \%$ de la population mondiale, mais ce chiffre est très vraisemblablement sous-estimé. L'OIM estime par exemple que les migrants irréguliers pourraient constituer 10 à $15 \%$ du total des migrants, soit entre 20 et 30 millions. Par ailleurs, il faut souligner que les statistiques sur les migrations sont souvent peu fiables et restent peu harmonisées entre les différents pays, y compris en Europe.

Les projections de migrations internationales sont difficiles, d'autant qu'il faut alors assurer une cohérence globale : les émigrés d'un pays doivent se retrouver dans les immigrés des autres pays. Par ailleurs, on ne dispose en général de statistiques ou d'estimations que sur la différence entre les nombres d'immigrés et d'émigrés, non sur chacune des composantes : la plupart des pays $\mathrm{n}^{\prime}$ enregistrent pas les sorties « définitives » de leur territoire. On mesure donc un « solde net », le plus souvent estimé à l'occasion des recensements.

Les hypothèses retenues dans les projections récentes des Nations unies sont très prudentes, probablement trop prudentes car le rythme des migrations semble plutôt s'accélérer. En effet, les taux de migrations nettes sont, pour la plupart des pays ou régions, plutôt inférieurs en 2040-50 à ceux de 2000 : en fait, ce sont les effectifs absolus de migrants (nets) qui sont maintenus constants tout au long de la projection, pour chacune des grandes régions du monde, et non les taux. L'Asie devrait perdre le plus grand nombre d'habitants (13 millions), suivie de l'Amérique latine (7) et de l'Afrique (5). Le nombre total de migrants net serait de l'ordre de 2 millions par an, soit 100 millions sur les 50 ans de projection. Mais ce nombre représente la différence entre les entrées et les sorties dans chaque région : le nombre réel de migrants devrait être deux à trois fois plus élevé.

À titre de comparaison, rappelons que sur un peu moins d'un siècle, de 1850 à 1930, environ 50 millions d'Européens ont migré outre-mer, dont la moitié aux États-Unis. $40 \%$ de ces émigrants ont quitté la Grande-Bretagne ou l'Italie, et $14 \%$ I'Irlande : pour ce dernier pays, le nombre total de migrants (7,3 millions) est proche de l'effectif de la population à la veille de la grande crise alimentaire de 1846-1850, dont I'Irlande ne se remettra jamais, plus de la moitié du flux migratoire ayant eu lieu après 1850 (Anderson et al., 1998). Les mouvements entre l'Europe et l'outre-mer ont donc été importants au cours de cette période, mais ils se sont faits principalement vers de grands espaces encore très peu peuplés (et dont on a d'ailleurs évincé les populations existantes). 
C'est dans le domaine des migrations que les effets démographiques des changements climatiques annoncés pourraient se faire le plus sentir. Deux causes principales sont envisagées : la désertification de certaines régions, en raison d'une baisse des précipitations, et la montée des eaux, couplée à une plus grande fréquence et une intensité accrue des cyclones ou tornades en bord de $\mathrm{mer}^{2}$ et aux débordements de rivières. Le réchauffement pourrait aussi rendre cultivables des zones actuellement stériles, et provoquer un "appel » de populations vers ces nouveaux territoires.

Rappelons d'abord qu'une migration de masse ne s'effectue pas toujours hors des frontières nationales, et quand $c^{\prime}$ est le cas le déplacement $s^{\prime}$ opère le plus souvent vers un pays voisin. $S^{\prime} i l n^{\prime}$ est pas organisé, ce type de mouvement peut engendrer de graves difficultés, d'abord alimentaires, mais aussi des conflits sérieux avec le pays "d'accueil » qui n'est pas toujours volontaire pour jouer ce rôle; on peut par exemple penser aux problèmes de voisinage entre le Bangladesh, particulièrement menacé par la montée des eaux, et l'Inde. Reste l'éventualité de mouvements à plus longue distance, comme de l'Afrique vers l'Europe, de certains pays d'Asie vers d'autres (de la Chine, voire du souscontinent indien, vers des terres du nord de la Sibérie, par exemple). La pression exercée actuellement aux frontières de l'Europe pourrait être le signe avantcoureur de tels mouvements, tout en en montrant la difficulté : les moyens de transport utilisés par ces clandestins n'ont que des capacités limitées, outre les risques considérables qu'ils font courir aux passagers. Il faudrait donc imaginer plutôt une migration voulue, organisée par les États concernés, dans un contexte d'intérêt réciproque (besoin de main d'œuvre dans les pays du Nord, par exemple, en raison du recul démographique).

La question plus spécifique de la montée des eaux est difficile ${ }^{3}$. Il est un fait que le peuplement tend à se concentrer le long des côtes, pour de multiples raisons : meilleures terres agricoles (deltas des fleuves...), géographie moins tourmentée, facilités des communications (par mer et sur les fleuves), etc. Des estimations de la population potentiellement exposée à la montée des eaux ont été proposées. On lit par exemple dans le dernier rapport du Giec (p. 414 du volume Contributions of Working Group II) qu'un quart de la population du monde vit à moins de $100 \mathrm{~km}$ du bord de la mer et à moins de $100 \mathrm{~m}$ d'altitude. Or la montée du niveau moyen des mers n'excède pas, dans les scénarios du Giec, $1 \mathrm{~m}$ : la référence aux « $100 \mathrm{~m}$ » semble donc peu utile, même en tenant compte des phénomènes d'entrée violente des eaux dans les territoires (vagues de plusieurs mètres).

\footnotetext{
${ }^{2}$ On pourrait ajouter les déplacements de populations induits par certains projets de développement, comme les grands barrages, ces projets étant impulsés par des déficits en eau dans d'autres régions.

${ }^{3}$ La montée du niveau des eaux entraîne une réduction des superficies cultivées et de l'habitat, mais affecte aussi l'ensemble des activités humaines liées à ces surfaces et habitats perdus.
} 
D'autres estimations ont été proposées. Elles divergent souvent sur l'ampleur de la montée des eaux. Le $4^{\mathrm{e}}$ rapport du Giec table sur une hausse de l'ordre de $60-70 \mathrm{~cm}$ en 2100, mais on considère généralement aujourd'hui que la vitesse de fonte des glaces du Groenland et antarctiques y était sous-estimée. Pfeffer et al. (2008) proposent une estimation minimale de $80 \mathrm{~cm}$, avec une fourchette allant jusqu'à $2 \mathrm{~m}$. Selon Dasgupta et al. (2007), 56 millions de personnes seraient impactées par une hausse de $1 \mathrm{~m}$ (dont 15 au Bangladesh), 89 millions avec une hausse de $2 \mathrm{~m}$, et 245 millions avec $5 \mathrm{~m}$. L'estimation de McGranahan et al. (2007) pour une montée de $10 \mathrm{~m}$ est en continuité avec ces valeurs : 634 millions de personnes concernées.

Il semble donc raisonnable de supposer que 50 à 100 millions de personnes, dans une hypothèse déjà pessimiste, pourraient être poussées à migrer en raison du réchauffement climatique, à l'horizon 2100. Cette dernière précision est essentielle : la montée des eaux sera progressive, et l'adaptation devra se faire sur plusieurs générations. Le chiffre de ces migrants forcés resterait nettement inférieur à celui des migrants "spontanés » (ils seront sûrement plus de 200 millions, d'après les projections faites sur 2000-2050) - sauf à envisager une montée des eaux océaniques beaucoup plus importante.

Il faudrait ajouter des migrations induites par la destruction des sols (désertification, érosion, etc.). Myers (2002) estime à 50 millions le nombre de personnes menacées par ces dégradations. Les événements climatiques extrêmes (inondations, cyclones, etc.) entraînent aussi des déplacements de population, partiellement réversibles : le tsunami de 2004 a déplacé 2 millions de personnes en Asie, I'ouragan Katrina 1,5 million de personnes en 2005 aux États-Unis. Lors des inondations catastrophiques de 1998 au Bangladesh, une grande partie du pays s'est trouvée (temporairement) sous l'eau et 30 millions de personnes ont perdu leur habitation. On peut envisager une augmentation de la fréquence de tels événements, qui frapperaient d'ailleurs en partie les zones inondables évoquées plus haut.

Redisons enfin que, contrairement à une idée reçue, les populations les plus vulnérables et les plus pauvres sont les moins susceptibles de migrer spontanément, en particulier de se déplacer en dehors des frontières nationales.

\section{Migrants et réfugiés}

\section{François Gemenne, Institut d'études politiques de Paris}

En premier lieu, les migrations volontaires doivent être distinguées des migrations forcées. Cette distinction, bien qu'elle paraisse simple, est en réalité malaisée : dans les flux migratoires se mêlent de plus en plus des personnes forcées à la fuite, et d'autres qui choisissent volontairement de partir. 
De surcroît, pour chaque migrant, les facteurs migratoires sont généralement divers et multiples, mêlant à la fois des éléments de coercition et des éléments de choix personnel. Cette distinction emporte pourtant des conséquences importantes, car les régimes internationaux de protection des migrants ne s'appliquent généralement qu'aux migrants forcés, et non aux migrants volontaires. Ces derniers restent aujourd'hui très peu protégés par le droit international. II existe aujourd'hui environ 16 millions de réfugiés dans le monde, dont 4 millions de réfugiés palestiniens. À ce chiffre, il faut ajouter 25 millions de personnes déplacées à l'intérieur de leur pays (IDPs), qui connaissent souvent des situations très semblables à celles des réfugiés, sans bénéficier de ce statut. La Convention de Genève de 1951 est supposée protéger au moyen du statut de réfugié ceux qui fuient leur pays à cause de persécutions liées à leur religion, leur nationalité, leur ethnicité, leurs opinions politiques ou leur appartenance à un groupe social particulier (un syndicat...), tandis que ceux qui fuient à l'intérieur de leur pays sont censés être protégés par les principes directeurs des Nations unies sur les déplacements internes, adoptés en 1998. Les réfugiés dits « climatiques » n'entrent donc pas dans la définition actuelle des réfugiés.

Une autre distinction concerne la légalité du processus migratoire. À côté des migrations qui s'effectuent dans le cadre prévu par la loi, un certain nombre de migrants entrent ou séjournent illégalement dans le pays d'accueil. Ils se placent dans l'illégalité en franchissant illégalement les frontières du pays, en y restant après l'expiration de leur visa, ou en effectuant un mariage blanc. L'Office international des migrations estime que les migrants irréguliers constituent entre 10 et $15 \%$ du total des migrants, soit entre 20 et 30 millions. Il est évidemment hasardeux par nature d'évoquer des estimations du nombre de migrants irréguliers, mais il semble que leur nombre soit en augmentation, suite au durcissement important des politiques migratoires dans la plupart des pays occidentaux.

Enfin, une dernière distinction naturelle, mais majeure, s'impose entre migrants internationaux et migrants internes. Le nombre de migrants internes n'est pas connu, mais on peut raisonnablement supposer qu'il dépasse celui des migrants internationaux. Ces migrations internes restent pourtant relativement peu connues, tant dans leurs dynamiques que dans leurs effets et leurs déterminants. Si beaucoup s'effectuent sur de relativement faibles distances, certaines sont bien plus distantes et ont un profond impact social c'est par exemple le cas des exodes ruraux, qui s'intensifient en Afrique. 


\section{2 | Risques et conséquences d'évolutions démographiques non prévues ${ }^{4}$}

Les projections habituelles, nous l'avons vu, n'intègrent pas d'hypothèses de discontinuités fortes. On pourrait imaginer divers scénarios : une épidémie particulièrement meurtrière et se répandant dans de nombreux pays ; un événement naturel exceptionnel et dévastateur; une crise alimentaire massive, en liaison peut-être avec le réchauffement climatique...

II meurt chaque année environ 55 millions de personnes, et l'excédent des naissances sur les décès est de l'ordre de 80 millions. Une catastrophe tuant ce nombre de personnes annulerait donc la croissance d'une année : à l'horizon 2050, cependant, elle n'affecterait qu'à peine la première décimale du nombre d'habitants en milliards. L'exemple le plus récent de grande épidémie mal contrôlée est celui de l'épidémie de sida : elle a tué environ 2 millions de personnes en 2007, et 25 millions en total cumulé depuis le début de l'épidémie. Une "grande famine » peut faire 1 million de morts; il faut remonter aux sombres épisodes de la Chine du Grand Bond en avant, de l'Union soviétique des années 1920 ou des grandes famines de I'Inde au milieu du XX siècle pour devoir compter le supplément de décès en dizaines de millions. Dans le cas de I'URSS, par exemple, une estimation assez précise du bilan des années 1918-1922 a été proposée récemment (Adamets, 2009) : environ 7 millions de décès liés aux épidémies, plus 2,4 parmi les militaires, et 7,8 en raison des famines et autres conséquences des troubles, le tout sur cinq ans et rapporté à une population totale d'environ 150 millions d'habitants (soit $11 \%$ de décès). Deux catastrophes récentes ayant fortement marqué les esprits, le tsunami de décembre 2004 en Asie et le tremblement de terre de Port-au-Prince en janvier 2010, ont fait chacune environ 200000 morts.

Deux événements ont été particulièrement dévastateurs au $X X^{e}$ siècle. La Seconde Guerre mondiale a fait entre 50 et 80 millions de victimes, civiles et militaires, sur 5 ans (la Première Guerre mondiale avait causé la mort de 10 à 13 millions de personnes). Quant à la terrible grippe espagnole de 1918-19, les estimations du nombre de décès vont de 40 à 100 millions (Séguy, 2009). On atteint là l'ordre de grandeur d'une année de croissance démographique actuelle, mais à l'époque cela représentait de 3 à 5 années de croissance. Indiquons aussi que sur l'ensemble du XXe siècle, les tremblements de terre ont fait entre 1 et 1,5 million de morts.

\footnotetext{
${ }^{4}$ Nous n'évoquerons pas ici certaines conséquences parfaitement prévisibles, comme le vieillissement des populations, ses relations avec la demande alimentaire et avec le changement climatique étant très limitées.
} 
Les périodes plus anciennes donnent des exemples de catastrophes importantes. Outre la disparition des populations précolombiennes d'Amérique, probablement due à l'importation de maladies européennes, on cite volontiers le cas de la peste en Europe au XIV siècle, parce qu'il est assez bien documenté : une succession d'épidémies aurait pu décimer un tiers de la population (Séguy, 2009). La maladie aurait en fait trouvé un terrain particulièrement propice, les populations concernées étant alors déjà en proie à des famines sévères et à $d^{\prime}$ autres causes de mortalité ; le même siècle a d'ailleurs été aussi marqué par une succession de conflits (la Guerre de cent ans). La peste ayant aussi frappé en Asie et en Afrique du Nord, son impact est visible sur la courbe de croissance de la population mondiale.

Il faut donc une catastrophe naturelle ou induite par l'homme particulièrement sévère pour qu'elle affecte significativement la croissance démographique mondiale.

Les auteurs de certaines projections démographiques ont tenté de prendre en compte un niveau d'incertitude sur l'évolution des paramètres. Il s'agit surtout de tenter de définir un intervalle d'évolution possible, pour la mortalité et la fécondité, cet intervalle pouvant éventuellement inclure des épisodes catastrophiques. Mais comme ces derniers sont supposés rares, ils $n^{\prime}$ influencent que faiblement l'intervalle de confiance. On voit donc la difficulté : ou bien l'on tient pour certaine l'occurrence d'un tel épisode au cours des 50 prochaines années, et son impact précis ne dépendra que de sa date; ou bien l'événement n'est affecté que d'une faible probabilité, et il est " noyé » dans la projection. II faut imaginer, en tout cas, une intensité extrêmement forte, donc probablement un impact simultané sur de larges régions du monde, pour que l'effet sur la population mondiale soit sensible.

On peut aussi évoquer une hypothèse rarement citée dans les scénarios des Nations unies : le maintien de l'espérance de vie à son niveau actuel pendant 50 ans, la fécondité évoluant selon l'hypothèse moyenne. La réduction de population attendue en 2050 serait forte, de l'ordre du milliard, mais avec une perte d'espérance de vie de 12 ans en fin de projection (64 ans au lieu des 76 attendus dans l'hypothèse centrale). Annuler un tel gain serait équivalent à l'annulation de tous les progrès réalisés dans la lutte contre la mortalité entre 1960 et 2000. Ce serait aussi équivalent à ce qui est projeté pour un des pays les plus atteints par l'épidémie de VIH-sida : le Zimbabwe. Dans ce pays de 12 millions d'habitants, I'espérance de vie était estimée à 61,4 ans en 1985-90. Après une chute spectaculaire sous l'effet du sida, elle devrait retrouver cette valeur vers 2025-30, soit après une quarantaine d'années.

Faudrait-il aussi considérer comme un autre type de "catastrophe » une baisse moins rapide de la fécondité, comme par exemple dans l'hypothèse 
haute des Nations unies (2,35 enfants en 2050), qui conduirait à 11 milliards d'hommes en 2050 ? Cette situation serait, de fait, difficile à gérer sur de multiples plans, à commencer par l'alimentation : comme le montrera ce rapport, nourrir 9 milliards d'hommes représentera déjà un défi difficile. Il faudra donc surveiller les tendances de la fécondité dans le monde, et réagir si l'on s'écarte sensiblement de la tendance centrale. Une telle divergence semblerait cependant encore moins probable dans un contexte de crise climatique, économique ou alimentaire.

Reste le scénario d'une catastrophe prolongée, telle que décrit par le Club de Rome en 1972 par exemple. Sous l'effet d'une crise alimentaire généralisée, la population mondiale se trouverait réduite de plusieurs milliards d'habitants en quelques dizaines d'années. Certains experts estiment ce scénario de plus en plus probable en raison du réchauffement climatique. L'un des buts du présent rapport est précisément de montrer comment il peut être évité, en se préparant à temps à faire face à la croissance démographique attendue tout en prenant en compte, autant que possible, les évolutions climatiques probables.

\section{Références bibliographiques}

Adamets S. (2009). Famines et épidémies dans les territoires de l'URSS entre 1918 et 1923. In : Vers une anthropologie des catastrophes (dir. par L. Buchet, C. Rigeade, I. Séguy et M. Signoli). Ed. APDCA et INED, Paris, pp. 237-249.

Anderson M., O'Grada C., Wrigley E.A., Woods R.I. (1998). Les îles Britaniques. In : Histoire des populations de l'Europe, tome II (éd. Par J.P. Bardet et J. Dupâquier). Fayard, Paris, pp. 327-347.

Dasgupta S., Laplante B., Meisner C., Wheeler D., Yan J. (2007). The impact of sea level rise on developing countries: a comparative analysis. In : World Bank Policy Research Working Paper $n^{\circ}$ 4136. The World Bank, Washington D.C.

IPCC (2007). Climate Change 2007. Synthesis Report. IPCC, Geneva.

IPCC (2008). Climate Change 2007: Impacts, Adaptation and Vulnerability (Contribution of Working Group II to the Fourth Assessment Report). Cambridge Univ. Press.

Li Jianmin (2002). La population de la Chine à l'horizon 2050. In : La Chine au seuil du XXI" siècle (dir. par I. Attané). INED, Paris, pp. 255-277. 
McGranahan G., Balk D., Anderson B. (2007). The rising tide: assessing the risks of climate change and human settlements in low elevation coastal zones. Environment and Urbanization, 19 (17) : 17-37.

Myers N. (2002). Environment and refugees: a growing phenomenon of the $21^{\text {st }}$ century. Phil. Trans. R. Soc. Lond. B, 357 : 609-613.

Pfeffer W.T., Harper J.T., O'Neel S. (2008). Kinematic constraints on glacier contributions to $21^{\text {st }}$ century sea-level rise. Science, 321 : 1340-43.

Séguy I. (2009). La catastrophe naturelle : le regard de l'historien-démographe. In: Vers une anthropologie des catastrophes (dir. par L. Buchet, C. Rigeade, I. Séguy et M. Signoli). Ed. APDCA et INED, Paris, pp. 77-97.

United Nations (2008). World Populations Prospects: the 2008 Revision. United Nations, New York.

United Nations (2008). World Urbanization Prospects: the 2007 Revision. United Nations, New York.

Véron J. (2006). Stabiliser la population de l'Inde : plus facile à dire qu'à faire. Populations et sociétés, $\mathbf{4 2 3}$, mai. 